\title{
Efficient Routability Check Algorithms for Segmented Channel Routing
}

\author{
CHENG-HSING YANG \\ Kung Shan Institute of Technology \\ SAO-JIE CHEN \\ National Taiwan University \\ JAN-MING HO \\ Academia Sinica \\ and \\ CHIA-CHUN TSAI \\ National Taipei University of Technology
}

The segmented channel-routing problem arises in the context of row-based field programmable gate arrays (FPGAs). Since the $K$-segment channel-routing problem is NP-complete for $K$ $\geq 2$, an efficient algorithm using the weighted bipartite-matching approach is developed for this problem. Connections that form a maximum clique are chosen first to be routed to the segmented channel. Then, another maximum clique of the remained connections is routed until all connections have been processed. In addition, a powerful "unroutability check" algorithm is uniquely proposed to tell whether the horizontal switches in an interval of the segmented channel are sufficient for routing or not. Hence, we can precisely discriminate the routable and the unroutable ones from all the test cases. As shown in the experiments, average discrimination ratios of $98.8 \%$ and $99.4 \%$ are obtained for the 2-segmentation and 3 -segmentation models, respectively. Moreover, when applying our routing algorithm to the analyzed nonunroutable cases, a routing failure ratio of $1.5 \%$ is reported for the 2 -segmentation model, compared to Zhu and Wong's 5.9\%; also, a routing failure ratio of $0.8 \%$ (less than their $4.7 \%$ ) is obtained for the 3-segmentation model. In total, the routing failure ratio of our routing algorithm is less than $21 \%$ of Zhu and Wong's.

Categories and Subject Descriptors: B.7.1 [Integrated Circuits]: Types and Design StylesGate arrays; B.7.2 [Integrated Circuits]: Design Aids-Placement and routing; J.6 [Computer Applications]: Computer-Aided Engineering-Computer-aided design (CAD)

This work was supported by the National Science Council R.O.C under grant NSC 86-2221E002-066 and NSC 86-2221-E027-003.

Authors' addresses: C.-H. Yang, Department of Information Management, Kung Shan Institute of Technology, Tainan, Taiwan; S.-J. Chen, Department of Electrical Engineering, National Taiwan University, Taipei, Taiwan; J.-M. Ho, Institute of Information Science, Academia Sinica, Taipei, Taiwan; C.-C. Tsai, Department of Electronic Engineering, National Taipei University of Technology, Taipei, Taiwan.

Permission to make digital/hard copy of part or all of this work for personal or classroom use is granted without fee provided that the copies are not made or distributed for profit or commercial advantage, the copyright notice, the title of the publication, and its date appear, and notice is given that copying is by permission of the ACM, Inc. To copy otherwise, to republish, to post on servers, or to redistribute to lists, requires prior specific permission and/or a fee.

(C) 2000 ACM 1084-4309/00/0700-0735 \$5.00 
General Terms: Algorithms, Design

Additional Key Words and Phrases: Field programmable gate arrays (FPGAs), routing, segmented channel

\section{INTRODUCTION}

The segmented-routing channels, or simply the segmented channels, in a row-based FPGA [El Gamal et al. 1989] consist of vertical and horizontal routing segments. Each input or output of the logic modules connects to a dedicated vertical segment; programmable switches are located at each crossing of the vertical and horizontal segments (cross switches) and also between pairs of adjacent horizontal segments on the same track (horizontal switches), such that the routing in a row-based FPGA can be performed by programming these switch elements [Greene et al. 1990; El Gamal et al. 1991]. Researches on how to design a segmented channel that maximizes the routability and satisfies performance requirements have been reported [Zhu and Wong 1992; Burman et al. 1992; Pedram et al. 1994], and this is denoted as a segmentation design problem.

A $K$-segment channel routing is a routing that assigns each connection to a track such that no segment is occupied by more than one connection and each connection occupies at most $K$ segments. Greene et al. [1990] state that the $K$-segment $(K>1)$ channel-routing problem is equivalent to the problem of numerical matching with target sums [Garey and Johnson 1979], and hence strongly NP-complete for $K \geq 2$. Exhaustive search [Greene et al. 1990; Roychowdhury et al. 1993]; bounded search [Roy 1993]; and heuristic algorithms [Zhu and Wong 1992] have been proposed to solve the K-segment channel-routing problem.

In this paper we develop a weighted bipartite-matching algorithm for the segmented channel-routing problem. We do not choose and assign connections one by one but clique by clique. The set of connections forming a maximum clique is chosen first. The routing of each clique of connections is done by finding a minimum weighted matching. If some connections cannot be assigned by the above step, postprocessing is used to reroute these connections. Also, in order to pick out the cases that are unroutable, we propose some criteria to check the unroutability of a case. Generally, given a set of connections to be routed over a range of columns, the number of tracks must be large enough such that each of the overlapping connections can be assigned to a different track, and the number of switches must also be sufficient such that as many nonoverlapped connections as possible can be assigned to the same track. Accordingly, a simple but powerful unroutability check method is proposed to tell whether the tracks and the switches between a range of columns are sufficient to complete the connections in this range.

The remainder of this paper is organized as follows: Section 2 describes some preliminary concepts and basic definitions. The routing and postpro- 
cessing algorithms are presented in Section 3. The unroutability check algorithm is stated in Section 4. Results on a set of benchmarks are reported in Section 5. Finally, a conclusion is drawn in Section 6.

\section{PRELIMINARIES AND DEFINITIONS}

In this paper we refer to Roychowdhury [1993] for the following definitions. The input to a segmented channel-routing problem, as depicted in Figure 1, is a segmented channel consisting of a set $\mathbf{T}$ of $T$ tracks and a set $\mathbf{C}$ of $M$ connections. A symbol $\bigcirc$ represents a horizontal switch and $\oplus$ represents a cross switch. Each track extending from column 1 to column $N$ is divided into a set of adjacent segments separated by horizontal switches.

For a segment $s$, left $(s)$ and right $(s)$ denote the leftmost and rightmost columns in which this segment is present. Similarly, a connection $c$ is uniquely characterized by the span of its leftmost and rightmost columns, left $(c)$ and right $(c)$. When a connection $c$ is assigned to a track $t$, the one or more segments in track $t$ that are present in the columns spanned by $c$ are considered occupied. More precisely, a segment $s$ in track $t$ is occupied by the connection $c$ if $\operatorname{right}(s) \geq \operatorname{left}(c)$ and $\operatorname{left}(s) \leq \operatorname{right}(c)$. For a $K$-segment channel routing, $c$ can be assigned to $t$ only if $c$ occupies at most $K$ segments. A connection $c$ is assignable (to a track $t$ ) if there exists such a track $t$ to which $c$ may be assigned and if the segments in track $t$ that are present in the columns spanned by $c$ are not occupied by another connection. Connections $c_{i}$ and $c_{j}$ overlap if their spans overlap. Connection $c_{i}$ collides with $c_{j}$ on a track $t$ if, when they are assigned to $t$, some of their occupied segments will overlap. For the segmented channel-routing problem, we restrict each connection that may be assigned to a single track only. The main objective of a $K$-segment channel routing is to find a full assignment of connections to tracks, i.e., to complete the assignment of connections to tracks such that each of the connections occupies at most $K$ segments. Another objective is to reduce the number of switches used in the routing.

Given a set $\mathbf{C}$ of $M$ connections with density $D$, which is the maximum number of connections over a column, a clique is a subset of $\mathbf{C}$ such that any $c_{i}$ and $c_{j}$ in it overlap each other. A maximal clique $\mathbf{Q}$ is a clique, in which there is no $c_{i} \notin \mathbf{Q}$ such that $\left\{c_{i}\right\} \cup \mathbf{Q}$ forms a new clique. A maximum clique is a maximal clique $\mathbf{Q}$ with the maximum cardinality, i.e., $|\mathbf{Q}|=D$. Note that any two connections in a clique cannot be assigned to the same track for they will occupy some same segments. A clique and the set of tracks form the two sets of nodes of a bipartite graph $G$. To find a full assignment of the clique $\mathbf{Q}$ is to find a maximum matching of the bipartite graph such that the cardinality of the maximum matching is equal to $|\mathbf{Q}|$. 


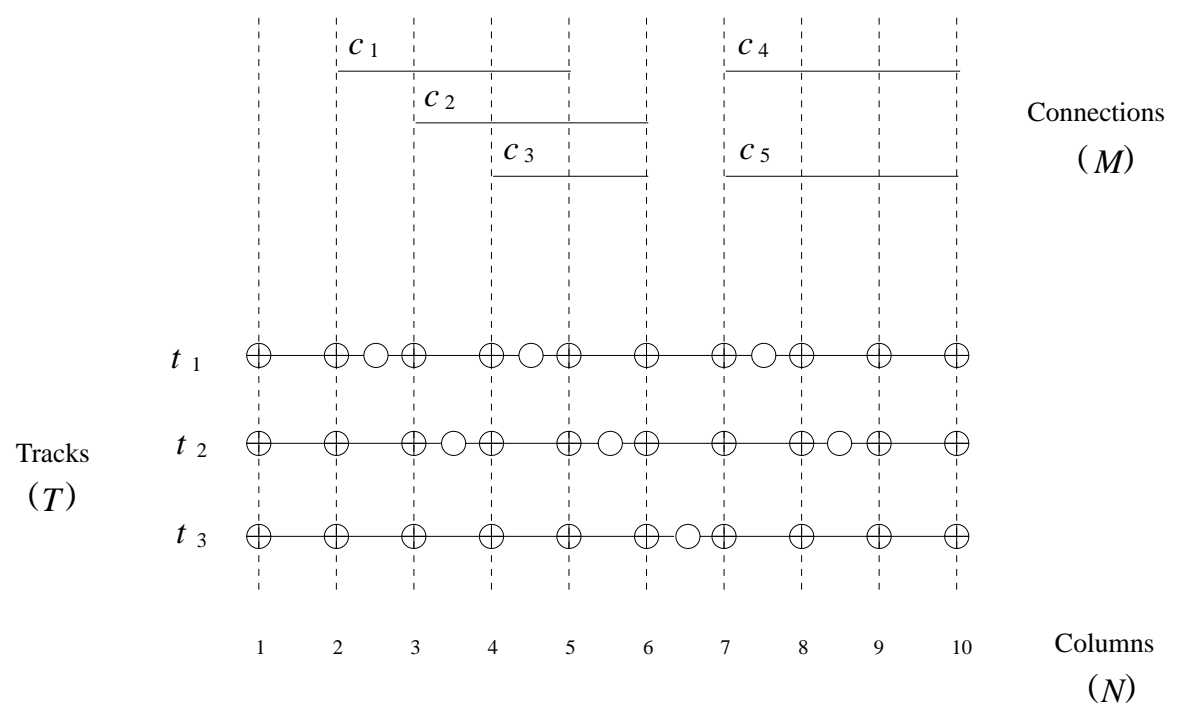

Fig. 1. The model of a segmented channel routing.

\section{THE ROUTING ALGORITHM}

To solve the segmented channel-routing problem efficiently, we apply the weighted bipartite matching algorithm to route and remove cliques of connections one by one. The weighted matching problem can be solved in time complexity $O\left(n^{3}\right)$ for a complete bipartite graph with $2 n$ nodes [Papadimitriou and Steiglitz 1982]. The routing algorithm is as follows:

\section{Algorithm. C_route $(\mathbf{C}, \mathbf{T})$}

$\{$ Do until $\mathbf{C}$ is empty:

$\{\mathbf{Q}=$ Remove_Max_Clique $(\mathbf{C})$;

$G=$ Construct_Bipartite_Graph $(\mathbf{Q}, \mathbf{T})$;

$($ Routed, Unrouted $)=$ Weighted_Bipartite_Matching $(G)$;

Total_Routed $=$ Total_Routed $\cup$ Routed;

\}

Total_Unrouted $=$ Total_Unrouted $\cup$ Unrouted;

if (Total_Unrouted is empty)

else return(feasible);

\}

return(Postprocessing(Total_Unrouted));

Every time a maximum clique $\mathbf{Q}$ of the remaining connections is picked out, a weighted bipartite graph for $\mathbf{Q}$ is constructed. The weight of edge $e_{i j}$ is defined as follows:

$$
\text { weight }\left(e_{i j}\right)= \begin{cases}\alpha \cdot w_{1}+\beta \cdot w_{2}+\gamma \cdot w_{3} & \text { if } c_{i} \text { is assignable to } t_{j} \\ \infty & \text { otherwise }\end{cases}
$$

where 


$$
\begin{array}{r}
\text { Efficient Routability } \\
w_{1}=\frac{\text { collision_number }\left(c_{i}, t_{j}\right)}{\text { remained_connection }(\mathbf{C})}, \\
w_{2}=\frac{\text { segment_length }\left(c_{i}, t_{j}\right)-\operatorname{connection\_ length~}\left(c_{i}\right)}{\operatorname{segment} \text { length }\left(c_{i}, t_{j}\right)},
\end{array}
$$

and

$$
w_{3}=\frac{\text { switch_number }\left(c_{i}, t_{j}\right)}{K-1} .
$$

In the above equations, collision_number $\left(c_{i}, t_{j}\right)$ is the number of remaining connections that collide with $c_{i}$ on track $t_{j}$; remained_connection $(\mathbf{C})$ is the total number of remaining connections in $\mathbf{C}$; segment_length $\left(c_{i}, t_{j}\right)$ is the total length of segments occupied by $c_{i}$ when $c_{i}$ is assigned to $t_{j}$; and connection_length $\left(c_{i}\right)$ is the length of $c_{i}$; switch_number $\left(c_{i}, t_{j}\right)$ is the number of switches that need to be programmed when $c_{i}$ is assigned to $t_{j} . \alpha, \beta$, and $\gamma$ are ratio parameters; $w_{1}$ denotes the degree of popularity on track $t_{j}$ for the remaining connections; and $w_{2}$ and $w_{3}$ denote the segment-length waste and switch usage, respectively. In order to save resources of segments and switches, a connection should be assigned to a track such that segment-length waste and switch usage are lower. Also, a connection should be assigned to a track with lower cost of $w_{1}$ such that fewer remaining connections will be affected. Weighted_ Bipartite_Matching finds a minimum weighted match. If connection $c_{i}$ is matched with track $t_{j}$ and weight $\left(e_{i j}\right) \neq \infty, c_{i}$ can be assigned to $t_{j}$ and is put into the Routed set. Otherwise, $c_{i}$ cannot be assigned and has to be put into the Unrouted set. The Total_Unrouted set is used to save all the connections not yet assigned. If Total_Unrouted is empty, this case is routable. Otherwise, a Postprocessing procedure is called to reroute the connections in Total_Unrouted again.

\section{Postprocessing}

When the routing of some connections cannot be completed in the routing step, we have to reroute them one-by-one using the Postprocessing step. But first we need some definitions. If a connection has been assigned to a track but is also assignable to another track, we say this connection is movable. When a connection $c_{i}$ is rerouted, it has to squeeze one connection $c_{j}$ assigned to a track $t$; that is, we have to remove $c_{j}$ from $t$ when assigning $c_{i}$ to $t$. Of course, this would only be done if the removal of $c_{j}$ implies that $c_{i}$ becomes assignable to $t$. If $c_{j}$ is movable, the rerouting of $c_{i}$ is done. Otherwise, $c_{j}$ has to squeeze another connection again. We do not consider the case where a connection would squeeze more than one connection on a 
track at a time, because this is too complex. The idea of squeezing can be implemented by the following algorithm.

\section{Algorithm. Postprocessing(Total_Unrouted)}

\{ Do until Total_Unrouted is empty:

$\{c=$ remove a connection from Total_Unrouted;

Queue $=\emptyset$;

Put all movable connections into Queue and mark these connections as roots;

Do while Queue is not empty and $c$ is not yet in Queue:

$\left\{c_{q}=\right.$ remove a connection from Queue;

$A=$ the connections that have never been in Queue and can squeeze $c_{q}$;

link all connections in $A$ to $c_{q}$;

\}

put all connections in $A$ into Queue;

if $c$ is not in Queue

return(infeasible);

else

reassign the connections in the squeeze path for $c$;

\}

return(feasible);

To reroute an unrouted connection $c$, all movable connections that become the roots of the squeeze paths are put into a Queue first. The connections, which have not been in Queue but can squeeze one connection $c_{q}$ in Queue, are linked to $c_{q}$ and put into Queue. If the unrouted connection $c$ is in Queue, the squeezing path for $c$ is found. Otherwise, $c$ cannot be rerouted by this approach.

\section{Time Complexity}

Procedures Construct_Bipartite_Graph, Weighted_Bipartite_Matching, and Postprocessing are the main parts of Algorithm $C_{-}$route. Let $q_{1}, q_{2}, \cdots$, $q_{m}$ be the cardinalities of cliques that are processed step by step in $C$ _route. For each step, say $q_{k}$, each weight $\left(e_{i j}\right)$ in Construct_Bipartite_Graph can be calculated at $O(M)$ time. There are $O\left(T^{2}\right)$ edges in a bipartite graph. Thus, Construct_Bipartite_Graph takes $O\left(M T^{2}\right)$ time. Also, Weighted_ Bipartite_Matching takes $O\left(T^{3}\right)$ time. So to process $m$ steps it takes $O\left(m M T^{2}+m T^{3}\right)$ time, which is less than $O\left(M^{2} T^{2}+M T^{3}\right)$.

Suppose $n$ connections need to be rerouted in Postprocessing. Each connection takes $O(M T)$ time to find the movable connections and to construct a squeeze path. Hence, Postprocessing takes $O(n M T)$ time, which is less than $O\left(M^{2} T\right)$. Thus the time complexity for Algorithm $C \_$route is $O\left(M^{2} T^{2}+M T^{3}\right)$. 


\section{Critical Path}

Our algorithm is designed for the $K$-segment channel routing problem. It does not consider the problem of critical path in a routing case. There are two possible methods of adjusting our algorithm to handle the problem of critical path. The first one is to route the connections in a critical path by first setting the $\gamma$ value to be much larger than the $\alpha$ and $\beta$ values. Then $C_{\text {_route is }}$ used to route the remaining connections if they are routable. The second method uses $C$ _route to route all connections as before, but the weight of each edge created by the connections in a critical path is set as follows: its $\gamma$ value must be larger than the $\alpha$ and $\beta$ values.

\section{UNROUTABILITY CHECK}

In the $C$ _route algorithm introduced in Section 3 , if the returned signal in $C_{\text {_route }}$ is feasible then, of course, this case is routable. But if C_route cannot find a feasible routing, it is not sufficient to say that it is unroutable. In this section we present the sufficient conditions for an unroutable case and develop an algorithm to check whether the case is really unroutable.

To describe the following lemmas, we assume that a $K$-segment channelrouting problem includes a set $\mathbf{C}$ of $M$ connections, a set $\mathbf{T}$ of $T$ tracks, and the density of $\mathbf{C}$ is $D$. Let $\mathbf{Q}$ be a clique of $\mathbf{C}$ and $G$ be the bipartite graph built from $\mathbf{Q}$ and $\mathbf{T}$. If the cardinality of the maximum matching for $G$ is equal to $|\mathbf{Q}|, \mathbf{Q}$ can be completely assigned. We call such a $G$ fully matchable.

Lemma 1. Given a clique $\boldsymbol{Q}$ of $\boldsymbol{C}$, if its bipartite graph $G$ is not fully matchable, $\boldsymbol{C}$ is unroutable.

Proof. $G$ not being fully matchable means that $\mathbf{Q}$ is unroutable. Because $\mathbf{Q} \subseteq \mathbf{C}, \mathbf{C}$ is unroutable.

Let $x=\operatorname{left}(c)$ and $\mathbf{Q}_{x}$ be the clique that consists of the connections spanning over column $x$, and $q_{x}=\left|\mathbf{Q}_{x}\right|$. As shown in Figure 2, when we scan the connections from column $x$ to the left until a certain column $x^{\prime}$, which is the rightmost column of a certain connection $c^{\prime}$, we find $k_{x}$ rightmost points appearing between $x-1$ and $x^{\prime}$, and these $k_{x}$ connections form a clique $\mathbf{Q}_{x^{\prime}}$. Suppose there exists a set $\mathbf{T}_{x}$ of $t_{x}$ tracks having switches between $x$ and $x^{\prime}$, we then have the following lemma.

Lemma 2. If $\boldsymbol{C}$ is routable, then $T-q_{x}+t_{x} \geq k_{x}$.

Proof. First, if each track in $T$ has no switch between $x-1$ and $x^{\prime}$, any two connections chosen from $\mathbf{Q}_{x}$ and $\mathbf{Q}_{x^{\prime}}$ will collide with each other on this track. In this case, if $\left|\mathbf{Q}_{x}\right|+\left|\mathbf{Q}_{x^{\prime}}\right|>T$, then $\mathbf{C}$ is unroutable. Second, since a track in $\mathbf{T}_{x}$ allows at most two connections, one from $\mathbf{Q}_{x}$ and the 
Connections

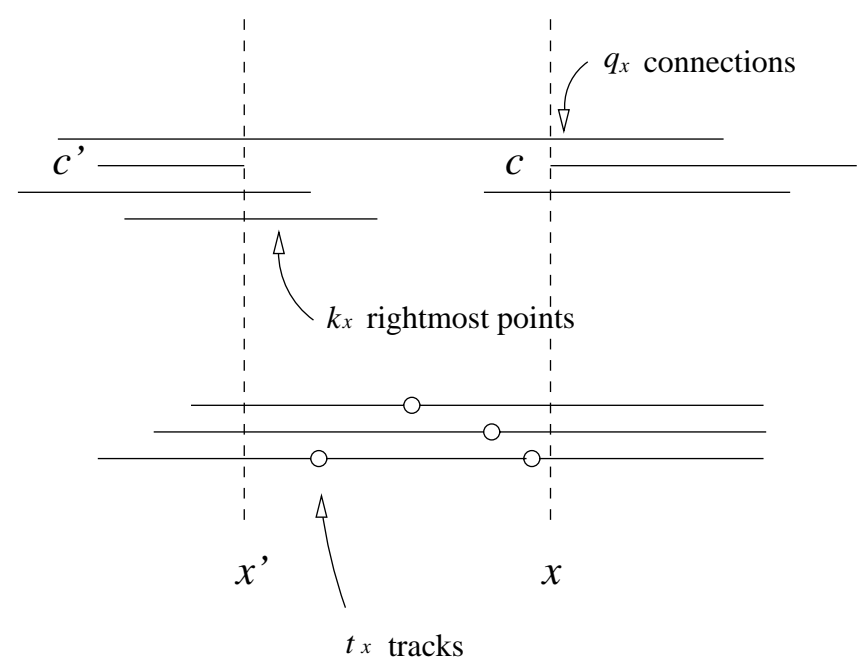

Fig. 2. Routability with the necessary condition $T-q_{x}+t_{x} \geq k_{x}$.

other from $\mathbf{Q}_{x^{\prime}}$, to be assigned, if $\left|\mathbf{Q}_{x}\right|+\left|\mathbf{Q}_{x^{\prime}}\right|-\left|\mathbf{T}_{x}\right|>T$, $\mathbf{C}$ is unroutable. Hence we have proven this lemma.

More precisely, let $m_{x}$ be the cardinality of the maximum matching for the bipartite graph built from $k_{x}$ connections and $t_{x}$ tracks. If $\mathbf{C}$ is routable, then $T-q_{x}+m_{x} \geq k_{x}$. Lemma 2 is also applicable to the case of column $x=\operatorname{right}(c)$, and the difference is that it scans the connections from column $x$ to the right and counts the connections whose leftmost points have been scanned.

Lemma 2 only considers the case where the $k_{x}$-counted connections form a clique. If some complete connections were to appear between $x-1$ and $x^{\prime}$, it may be the case that two counted connections were assigned to the same track. To deal with this case, we have to change Lemma 2 by considering the following two conditions. Condition (1): there is no complete segment that appears between $x-1$ and $x^{\prime}$. Condition (2): there exist complete segments that appear between $x-1$ and $x^{\prime}$. Considering Condition (1), since all counted connections cannot be assigned to the same track in $\mathbf{T}_{x}$, Lemma 2 is still suitable. Considering Condition (2), we have to partition $k_{x}$ connections into two parts: one consisting of $k_{x_{1}}$ connections that do not appear complete between $x-1$ and $x^{\prime}$; the other consists of $k_{x_{2}}$ connections that appear complete between $x-1$ and $x^{\prime}$. Let $s_{x}$ be the number of segments that appear complete between $x-1$ and $x^{\prime}$. In order to check unroutability, we need to know that how many $k_{x_{2}}$ connections can be assinged to the $s_{x}$ segments. This problem is similar to the $K$-segment channel-routing problem, and thus is NP-complete. However, we can calculate the maximum number of connections to be assigned to each track, which is formed by these $s_{x}$ segments independently. Then, we obtain an upper bound of the above problem by totalizing these maximum numbers. 
The maximum number can be calculated easily if we sort in increasing order the $k_{x_{2}}$ connections by their rightmost columns, and then assign them to a track according to the sorting order. Let the total be $s^{\prime}{ }_{x}$ and

$$
k_{x_{2}}^{\prime}= \begin{cases}k_{x_{2}}-s^{\prime}{ }_{x} & \text { if } k_{x_{2}}>s^{\prime}{ }_{x} \\ 0 & \text { if } k_{x_{2}} \leq s^{\prime}{ }_{x} .\end{cases}
$$

Thus, there are at least $k_{x_{1}}+k_{x_{2}}^{\prime}$ counted connections that cannot be assigned to the same track in $\mathbf{T}_{x}$. Note that Condition (1) is a special case of Condition (2) for $s_{x}=0$. Now we have the following lemma:

LEMMA 3. If $\boldsymbol{C}$ is routable, then $T-q_{x}+t_{x} \geq k_{x_{1}}+k_{x_{2}}^{\prime}$.

Given connections $\mathbf{C}$ and tracks $\mathbf{T}$, the Unroutability_check algorithm uses Lemma 3 to check whether $\mathbf{C}$ is unroutable. The cardinalities of each clique of connections $\mathbf{Q}_{x}$ can be calculated column by column in linear time if connections in $\mathbf{C}$ are sorted [Rheinboldt 1980]. In order to save time, only the columns whose $\left|\mathbf{Q}_{x}\right|$ is larger than ( $D-$ CLIQUE_CONSTANT) are processed. In our experiments, almost no unroutable cases are found when CLIQUE_CONSTANT is set to 8. Scanning from column $x$ to the left, whenever a connection $c^{\prime}$ and its rightmost column $x^{\prime}$ is found, Lemma 3 is used to check the unroutability. If there exist $x$ and $x^{\prime}$ such that $T-q_{x}$ $+t_{x}<k_{x_{1}}+k_{x_{2}}^{\prime}, \mathbf{C}$ is unroutable. Otherwise, Algorithm Unroutability check fails to check whether $\mathbf{C}$ is unroutable.

\section{Algorithm. Unroutability_check $(\mathbf{C}, \mathbf{T})$}

$\left\{\right.$ To calculate $\left|\mathbf{Q}_{x}\right|$ of each column $x$ for $\mathbf{C}$;

For each column $x$ such that $\left|\mathbf{Q}_{x}\right|>(D-$ CLIQUE_CONSTANT) do:

$\left\{\right.$ if $\left(\mathbf{Q}_{x}\right.$ is a maximal clique and $x$ is the leftmost column of a certain connection)

scan from $x$ to the left to find $x^{\prime}$ columns and use Lemma 3 to check unroutability;

if $\left(\mathbf{Q}_{x}\right.$ is a maximal clique and $x$ is the rightmost column of a certain connection)

scan from $x$ to the right to find $x^{\prime}$ columns and use Lemma 3 to check unroutability;

\}

if (one of above checks does not satisfy Lemma 3)

else return(unroutable);

\}

return(fail to check);

For each column $x$ and maximal clique $\mathbf{Q}_{x}$, it takes $O(M T)$ time to assign $k_{x_{2}}$ connections to each of $t_{x}$ tracks after a column $x^{\prime}$ is found. Since there are at most $O(M)$ of maximal cliques and the number of $x^{\prime}$ columns that each maximal clique $\mathbf{Q}_{x}$ needs to scan is $O(M)$, the time complexity of Algorithm Unroutability check is $O\left(M^{3} T\right)$. 
Table I. Routability Analysis Results for the $K$-Segmentation Models

\begin{tabular}{|c|c|c|c|c|c|c|c|c|c|c|}
\hline \multirow{3}{*}{$\begin{array}{c}\text { Segmented } \\
\text { channel }\end{array}$} & \multicolumn{4}{|c|}{ C_route } & \multicolumn{4}{|c|}{ Unroutability_check } & \multicolumn{2}{|c|}{ DR } \\
\hline & \multicolumn{2}{|c|}{$K=2$} & \multicolumn{2}{|c|}{$K=3$} & \multicolumn{2}{|c|}{$K=2$} & \multicolumn{2}{|c|}{$K=3$} & \multirow[t]{2}{*}{$K=2$} & \multirow[t]{2}{*}{$K=3$} \\
\hline & $\mathrm{RC}$ & sec. & $\mathrm{RC}$ & sec. & UC & sec. & $\mathrm{UC}$ & sec. & & \\
\hline D1 & 207 & 61.8 & 185 & 68.1 & 92 & 9.5 & 115 & 10.1 & $99.7 \%$ & $100.0 \%$ \\
\hline D2 & 258 & 54.2 & 257 & 60.9 & 42 & 3.5 & 43 & 3.6 & $100.0 \%$ & $100.0 \%$ \\
\hline D3 & 203 & 76.3 & 186 & 88.5 & 95 & 30.0 & 113 & 28.6 & $99.3 \%$ & $99.7 \%$ \\
\hline $\mathrm{D} 4$ & 211 & 98.1 & 203 & 119.9 & 80 & 74.6 & 94 & 67.6 & $97.0 \%$ & $99.0 \%$ \\
\hline D5 & 174 & 68.1 & 180 & 72.8 & 126 & 8.1 & 120 & 12.1 & $100.0 \%$ & $100.0 \%$ \\
\hline D6 & 250 & 49.6 & 211 & 60.6 & 50 & 10.2 & 89 & 7.2 & $100.0 \%$ & $100.0 \%$ \\
\hline D7 & 208 & 180.0 & 217 & 201.5 & 87 & 188.1 & 78 & 186.1 & $98.3 \%$ & $98.3 \%$ \\
\hline Geometric & 164 & 169.5 & 165 & 188.1 & 133 & 82.7 & 135 & 83.9 & $99.0 \%$ & $100.0 \%$ \\
\hline Normal & 267 & 47.8 & 292 & 62.6 & $23^{*}$ & 33.6 & 4 & 27.1 & $96.7 \%$ & $98.7 \%$ \\
\hline Poisson & 250 & 140.1 & 250 & 152.6 & 45 & 174.0 & 44 & 167.0 & $98.3 \%$ & $98.0 \%$ \\
\hline Average & 219 & 94.6 & 214 & 107.3 & 77 & 44.9 & 84 & 59.3 & $98.8 \%$ & $99.4 \%$ \\
\hline
\end{tabular}

\section{EXPERIMENTAL RESULTS}

Our C_route and Unroutability_check algorithms were implemented in the $\mathrm{C}$ language and tested on the 300 set connections created by Zhu and Wong [1992]. All experiments ran on a SUN Sparc 10 workstation with $32 \mathrm{MB}$ main memory. The parameters of the channel model, $N=100$ and $T=$ 36, are close to the Actel's ACT2 family A1280 FPGA [Actel Corporation 1991]. The ratio parameters $\alpha, \beta$, and $\gamma$ in our experiments are $0.5,0.4$, and 0.1 , respectively. Zhu and Wong's 2-segmentation models and 3-segmentation models were used; ten types of connection distributions, D1 through D7, geometric, normal, and Poisson, were tested. Table I shows the 2 -segment and 3-segment channel-routability analysis results using C_route and Unroutability_check on each distribution of 300 test cases. RC is the number of cases that can feasibly be routed by $C$ _route. UC is the number of cases that can be checked as unroutable by Unroutability_check. The total time to run the 300 cases for each distribution is also shown in the table. The last two columns show the discrimination ratios obtained from the results of these two algorithms, i.e., the ratio of $\mathrm{RC}+\mathrm{UC}$ cases over 300 cases for each distribution. The discrimination ratio is denoted as DR. The average discrimination ratios are $98.8 \%$ and $99.4 \%$ for 2 -segment and 3-segment channel routing, respectively.

Table II and Table III compare the 2-segment channel-routing and 3-segment channel-routing results, respectively, of Zhu and Wong's routing algorithm with our $C$ _route algorithm. Column 2 shows the number of test cases, excluding the UC cases for each distribution. FC is the number of cases that fail to be routed. The failure ratios of our routing algorithm are all less than or equal to those of Zhu and Wong's. For the 2-segment channel routing, ours is less than $25 \%$ of theirs on the average. For the 3 -segment channel routing, ours is less than $17 \%$ of theirs on the average. The disadvantage of our approach is that our runtime is longer than theirs. 
Table II. Comparing Zhu and Wong's 2-Segment Channel Routing Algorithm with Others

\begin{tabular}{|c|c|c|c|c|c|c|c|}
\hline \multirow{2}{*}{$\begin{array}{c}\text { Segmented } \\
\text { channel }\end{array}$} & \multirow{2}{*}{$\begin{array}{l}\text { No. of } \\
\text { cases }\end{array}$} & \multicolumn{2}{|c|}{ Zhu and Wong } & \multicolumn{2}{|c|}{ C_Route } & \multicolumn{2}{|c|}{ Failure ratio } \\
\hline & & $\mathrm{FC}$ & sec. & $\mathrm{FC}$ & sec. & Zhu and Wong & C_route \\
\hline D1 & 208 & 8 & 4.5 & 1 & 33.4 & $3.8 \%$ & $0.5 \%$ \\
\hline D2 & 258 & 1 & 3.3 & 0 & 43.0 & $0.4 \%$ & $0.0 \%$ \\
\hline D3 & 205 & 9 & 9.0 & 2 & 35.2 & $4.4 \%$ & $1.0 \%$ \\
\hline D4 & 220 & 21 & 15.3 & 9 & 49.0 & $9.5 \%$ & $4.1 \%$ \\
\hline D5 & 174 & 5 & 4.4 & 0 & 26.5 & $2.9 \%$ & $0.0 \%$ \\
\hline D6 & 250 & 11 & 4.4 & 0 & 36.8 & $4.4 \%$ & $0.0 \%$ \\
\hline D7 & 213 & 30 & 23.0 & 5 & 77.0 & $14.1 \%$ & $2.3 \%$ \\
\hline Geometric & 167 & 11 & 14.7 & 3 & 50.1 & $6.6 \%$ & $1.8 \%$ \\
\hline Normal & 277 & 17 & 8.2 & 10 & 41.1 & $6.1 \%$ & $3.6 \%$ \\
\hline Poisson & 255 & 17 & 24.4 & 5 & 91.6 & $6.7 \%$ & $2.0 \%$ \\
\hline Average & 223 & 13 & 11.1 & 4 & 48.4 & $5.9 \%$ & $1.5 \%$ \\
\hline
\end{tabular}

Table III. Comparing Zhu and Wong's Algorithm on 3-Segment Channel Routing

\begin{tabular}{|c|c|c|c|c|c|c|c|}
\hline \multirow{2}{*}{$\begin{array}{c}\text { Segmented } \\
\text { channel }\end{array}$} & \multirow{2}{*}{$\begin{array}{l}\text { No. of } \\
\text { cases }\end{array}$} & \multicolumn{2}{|c|}{ Zhu and Wong } & \multicolumn{2}{|c|}{ C_Route } & \multicolumn{2}{|c|}{ Failure ratio } \\
\hline & & $\mathrm{FC}$ & sec. & $\mathrm{FC}$ & sec. & Zhu and Wong & C_route \\
\hline D1 & 185 & 0 & 5.3 & 0 & 30.7 & $0.0 \%$ & $0.0 \%$ \\
\hline D2 & 257 & 0 & 4.5 & 0 & 47.3 & $0.0 \%$ & $0.0 \%$ \\
\hline D3 & 187 & 21 & 12.9 & 1 & 35.3 & $11.2 \%$ & $0.5 \%$ \\
\hline D4 & 206 & 7 & 17.7 & 3 & 50.9 & $3.4 \%$ & $1.5 \%$ \\
\hline D5 & 180 & 0 & 5.5 & 0 & 30.2 & $0.0 \%$ & $0.0 \%$ \\
\hline D6 & 211 & 10 & 5.2 & 0 & 34.9 & $4.7 \%$ & $0.0 \%$ \\
\hline D7 & 222 & 28 & 34.4 & 5 & 93.3 & $12.6 \%$ & $2.3 \%$ \\
\hline Geometric & 165 & 12 & 20.4 & 4 & 50.4 & $7.3 \%$ & $0.0 \%$ \\
\hline Normal & 296 & 9 & 10.6 & 4 & 60.7 & $3.0 \%$ & $1.4 \%$ \\
\hline Poisson & 256 & 13 & 28.6 & 6 & 101.1 & $5.1 \%$ & $2.3 \%$ \\
\hline Average & 217 & 10 & 14.5 & 2 & 53.5 & $4.7 \%$ & $0.8 \%$ \\
\hline
\end{tabular}

The results of postprocessing are shown in Table IV. Columns 3 to 6 show the number of cases routed with the help of postprocessing for Zhu and Wong's and our algorithms. The ratios are the numbers of routed cases over the cases numbers shown in Table II and Table III. Although the benefit obtained by postprocessing is small, it is helpful when the failure ratio of the main routing algorithm is quite small also. This also means that our weighted bipartite matching approach is efficient.

We speculated that our routing algorithm would work badly if $\mathrm{N}$ were larger than the average length of the connections. So we tested our algorithm for the channel models using different $\mathrm{N}$ values. First, we used Zhu and Wong's program to generate 3-segmentation models for $\mathrm{N}=100$, $200, \cdots, 2000$. The results for $\mathrm{N}=2000$ are shown in Table $\mathrm{V} . \mathrm{RC}_{1}$ and $\mathrm{RC}_{2}$ are the numbers of cases which can feasibly be routed by, respectively, Zhu and Wong's algorithm and our $C_{-}$route. UC is the number of cases that can be checked unroutable by Unroutability_check. DR is the discrimination ratio. $\mathrm{DF}$ is the difference for the failure ratio, which is the value of Zhu and Wong's failure ratio minus our failure ratio. Our C_route is still 
Table IV. Results of Postprocessing on the $K$-Segmentation Models

\begin{tabular}{|c|c|c|c|c|c|c|c|c|}
\hline \multirow{3}{*}{$\begin{array}{c}\text { Segmented } \\
\text { channel }\end{array}$} & \multicolumn{4}{|c|}{ Routed by postprocessing } & \multicolumn{4}{|c|}{ Ratio } \\
\hline & \multicolumn{2}{|c|}{ Zhu and Wong } & \multicolumn{2}{|c|}{ C_route } & \multicolumn{2}{|c|}{ Zhu and Wong } & \multicolumn{2}{|c|}{ C_route } \\
\hline & $K=2$ & $K=3$ & $K=2$ & $K=3$ & $K=2$ & $K=3$ & $K=2$ & $K=3$ \\
\hline D1 & 0 & 0 & 0 & 0 & $0.0 \%$ & $0.0 \%$ & $0.0 \%$ & $0.0 \%$ \\
\hline D2 & 0 & 0 & 0 & 0 & $0.0 \%$ & $0.0 \%$ & $0.0 \%$ & $0.0 \%$ \\
\hline D3 & 0 & 0 & 0 & 0 & $0.0 \%$ & $0.0 \%$ & $0.0 \%$ & $0.0 \%$ \\
\hline $\mathrm{D} 4$ & 1 & 1 & 8 & 1 & $0.5 \%$ & $0.5 \%$ & $3.6 \%$ & $0.5 \%$ \\
\hline D5 & 0 & 0 & 0 & 0 & $0.0 \%$ & $0.0 \%$ & $0.0 \%$ & $0.0 \%$ \\
\hline D6 & 0 & 0 & 0 & 0 & $0.0 \%$ & $0.0 \%$ & $0.0 \%$ & $0.0 \%$ \\
\hline D7 & 12 & 3 & 12 & 8 & $5.6 \%$ & $1.4 \%$ & $5.6 \%$ & $3.6 \%$ \\
\hline Geometric & 0 & 0 & 2 & 4 & $0.0 \%$ & $0.0 \%$ & $1.2 \%$ & $2.4 \%$ \\
\hline Normal & 3 & 1 & 6 & 0 & $1.1 \%$ & $0.3 \%$ & $2.2 \%$ & $0.0 \%$ \\
\hline Poisson & 0 & 0 & 2 & 2 & $0.0 \%$ & $0.0 \%$ & $0.8 \%$ & $0.8 \%$ \\
\hline Average & 1.6 & 0.5 & 3.0 & 1.5 & $0.7 \%$ & $0.2 \%$ & $1.3 \%$ & $0.7 \%$ \\
\hline
\end{tabular}

Table V. Routability Analysis for the 3-Segmentation Models with $\mathrm{N}=2000$

\begin{tabular}{ccccrrr}
\hline $\begin{array}{c}\text { Segmented } \\
\text { channel }\end{array}$ & $\begin{array}{c}\text { Average } \\
\text { connection } \\
\text { length }\end{array}$ & $\mathrm{RC}_{1}$ & $\mathrm{RC}_{2}$ & $\mathrm{UC}$ & $\mathrm{DR}$ & $\mathrm{DF}$ \\
\hline $\mathrm{D} 1$ & 986 & 173 & 173 & 127 & $100.0 \%$ & $0.0 \%$ \\
$\mathrm{D} 2$ & 1334 & 249 & 249 & 51 & $100.0 \%$ & $0.0 \%$ \\
D3 & 646 & 140 & 152 & 147 & $99.7 \%$ & $7.8 \%$ \\
D4 & 483 & 192 & 204 & 90 & $98.0 \%$ & $5.7 \%$ \\
D5 & 978 & 187 & 186 & 113 & $99.7 \%$ & $-0.5 \%$ \\
D6 & 989 & 219 & 238 & 62 & $100.0 \%$ & $8.0 \%$ \\
D7 & 319 & 162 & 172 & 126 & $99.3 \%$ & $5.7 \%$ \\
Geometric & 20 & 130 & 137 & 140 & $92.3 \%$ & $4.4 \%$ \\
Normal & 700 & 279 & 284 & 7 & $97.0 \%$ & $1.7 \%$ \\
Poisson & 20 & 111 & 115 & 172 & $95.7 \%$ & $3.1 \%$ \\
Average & 628 & 184 & 191 & 104 & $98.2 \%$ & $3.6 \%$ \\
\hline
\end{tabular}

efficient and generates better results than Zhu and Wong's for this large $\mathrm{N}$ value. Some of results with different $\mathrm{N}$ values are shown in Table VI. The averages of DF are always at least 3\% larger than Zhu and Wong's. And the averages of DR are all larger than $97 \%$.

\section{CONCLUSIONS}

We presented a weighted bipartite-matching algorithm for the segmented channel-routing problem. Our approach routes the connections clique by clique, where the connections in each clique are routed using the weighted bipartite-matching method. An approach to successfully judging unroutability is also presented. The average discrimination ratios of the 300 routing cases are $98.8 \%$ and $99.4 \%$ for the 2 -segmentation and 3 -segmentation models, respectively. Applying our segmented channel-routing algorithm to the nonunroutable cases, the average of our failure ratios is less than $21 \%$ of Zhu and Wong's algorithm. 
Table VI. Routability Analysis for the 3-Segmentation with Different N Values

\begin{tabular}{|c|c|c|c|c|c|c|c|c|}
\hline \multirow{2}{*}{$\begin{array}{c}\text { Segmented } \\
\text { channel }\end{array}$} & \multicolumn{2}{|c|}{$\mathrm{N}=400$} & \multicolumn{2}{|c|}{$\mathrm{N}=800$} & \multicolumn{2}{|c|}{$\mathrm{N}=1200$} & \multicolumn{2}{|c|}{$\mathrm{N}=1600$} \\
\hline & DR & $\mathrm{DF}$ & DR & $\mathrm{DF}$ & DR & $\mathrm{DF}$ & DR & $\mathrm{DF}$ \\
\hline D1 & $100.0 \%$ & $0.0 \%$ & $100.0 \%$ & $0.0 \%$ & $100.0 \%$ & $0.0 \%$ & $100.0 \%$ & $0.0 \%$ \\
\hline D2 & $100.0 \%$ & $0.0 \%$ & $100.0 \%$ & $0.0 \%$ & $100.0 \%$ & $0.4 \%$ & $100.0 \%$ & $0.0 \%$ \\
\hline D3 & $100.0 \%$ & $3.2 \%$ & $100.0 \%$ & $6.3 \%$ & $99.7 \%$ & $4.2 \%$ & $99.3 \%$ & $3.5 \%$ \\
\hline D4 & $99.3 \%$ & $2.5 \%$ & $99.3 \%$ & $2.5 \%$ & $99.3 \%$ & $1.2 \%$ & $99.7 \%$ & $3.3 \%$ \\
\hline D5 & $100.0 \%$ & $0.5 \%$ & $100.0 \%$ & $0.5 \%$ & $99.7 \%$ & $0.0 \%$ & $100.0 \%$ & $0.0 \%$ \\
\hline D6 & $99.0 \%$ & $21.4 \%$ & $99.3 \%$ & $11.7 \%$ & $99.7 \%$ & $13.3 \%$ & $99.0 \%$ & $17.3 \%$ \\
\hline D7 & $98.3 \%$ & $8.5 \%$ & $98.7 \%$ & $7.2 \%$ & $97.7 \%$ & $8.9 \%$ & $98.7 \%$ & $2.7 \%$ \\
\hline Geometric & $97.7 \%$ & $5.9 \%$ & $99.3 \%$ & $2.7 \%$ & $91.3 \%$ & $5.6 \%$ & $94.0 \%$ & $5.1 \%$ \\
\hline Normal & $99.3 \%$ & $3.1 \%$ & $98.0 \%$ & $3.8 \%$ & $97.0 \%$ & $4.4 \%$ & $96.7 \%$ & $2.4 \%$ \\
\hline Poisson & $97.3 \%$ & $7.2 \%$ & $93.0 \%$ & $3.2 \%$ & $95.0 \%$ & $-0.6 \%$ & $94.7 \%$ & $4.2 \%$ \\
\hline Average & $99.1 \%$ & $5.3 \%$ & $98.8 \%$ & $3.8 \%$ & $97.9 \%$ & $3.7 \%$ & $98.2 \%$ & $3.8 \%$ \\
\hline
\end{tabular}

\section{REFERENCES}

ACtel CorP. 1991. ACT Family FPGA Databook.

Burman, S., Kamalanathan, C., And Sherwani, N. 1992. New channel segmentation model and associated routing algorithm for high performance FPGAs. In Proceedings of the 1992 IEEE/ACM International Conference on Computer-Aided Design (ICCAD '92, Santa Clara, CA, Nov. 8-12), L. Trevillyan, Ed. IEEE Computer Society Press, Los Alamitos, CA, 22-25.

El Gamal, A., Greene, J., And Roychowdhury, V. 1991. Segmented channel routing in nearly as efficient as channel routing (and just as hard). In Proceedings of the 1991 University of California / Santa Cruz Conference on Advanced Research in VLSI (Edimburgh, Scotland), C. H. Séquin, Ed. MIT Press, Cambridge, MA, 192-211.

El Gamal, A., Greene, J., Reynari, J., Rogoyski, E., El Ayat, K. A., And Mohsen, A. 1989. An architecture for electrically configurable gate arrays. IEEE J. Solid-State Circuits 24, 2 (Apr.), 394-398.

Garey, M. And Johnson, D. 1979. Computers and Intractability: A Guide to the Theory of NP-Completeness. W. H. Freeman and Co., New York, NY.

Greene, J., Roychowdhury, V., Kaptanoglu, S., And Gamal, A. E. 1990. Segmented channel routing. In Proceedings of the ACM/IEEE Conference on Design Automation (DAC '90, Orlando, FL, June 24-28), R. C. Smith, Ed. ACM Press, New York, NY, 567-572.

Papadimitriou, C. H. And Steiglitz, K. 1982. Combinatorial Optimization: Algorithms and Complexity. Prentice-Hall, Inc., Upper Saddle River, NJ.

Pedram, M., Nobandegani, B. S., And Preas, B. T. 1994. Design and analysis of segmented routing channels for row-based FPGA's. IEEE Trans. Comput.-Aided Des. 13, 12 (Dec.), $1470-1479$.

Rheinboldt, W. 1980. Algorithmic Graph Theory and Perfect Graphs. Academic Press, Inc., New York, NY.

RoY, K. 1993. A bounded search algorithm for segmented channel routing for FPGA's and associated channel architecture issues. IEEE Trans. Comput.-Aided Des. 12, 11, 1695-1705.

Roychowdhury, V. P., Greene, J., AND El Gamal, A. 1993. Segmented channel routing. IEEE Trans. Comput.-Aided Des. 12, 1, 79-95.

ZhU, K. And Wong, D. F. 1992. On channel segmentation design for row-based FPGAs. In Proceedings of the 1992 IEEE/ACM International Conference on Computer-Aided Design (ICCAD '92, Santa Clara, CA, Nov. 8-12), L. Trevillyan, Ed. IEEE Computer Society Press, Los Alamitos, CA, 26-29.

Received: July 1997; accepted: March 1999 